\title{
Evaluating the analgesic effect and advantage of transcutaneous electrical acupoint stimulation combined with opioid drugs for moderate to severe cancer- related pain: a study protocol for a randomized controlled trial
}

Yi Liang ${ }^{1,2+}$, Guanai Bao ${ }^{3+}$, Liyan Gong ${ }^{3}$, Jie Zhou' ${ }^{1}$ Xiangming Kong ${ }^{3}$, Ran Ran', Xiaomei Shao ${ }^{1,2}$, Yongliang Jiang ${ }^{1,2}$, Weiping Zhang ${ }^{1}$, Boyi Liu ${ }^{2}$, Junying Du ${ }^{1,2}$, Junfan Fang ${ }^{1,2}, \mathrm{Na} \mathrm{Nie}^{1}$, Conghua $\mathrm{Ji}^{4}$ and Jianqiao Fang ${ }^{1,2^{*}}$

\begin{abstract}
Background: Transcutaneous electrical acupoint stimulation (TEAS), which is also known as acupuncture-like transcutaneous electrical nerve stimulation (TENS), has been widely used in acute or chronic pain. However, previous research has not demonstrated that TEAS is effective for cancer-related pain. Opioid drugs are strongly recommended for treating cancer-related pain, but opioid-induced immunosuppression is still the most intractable drug-induced medical problem. Evaluating the efficacy and potential advantage of TEAS combined with opioid drugs in moderate and severe cancer-related pain in China is important because such studies are lacking.

Methods/Design: This trial is a multicenter, prospective randomized controlled clinical trial. In total, 160 patients who were enrolled from two hospitals in the Zhejiang Province (China) will be randomly allocated into two groups: a TEAS group and sham TEAS group without acupoint electrical stimulation. Both groups will receive a 21-day interval of chemotherapy and conventional cancer pain therapy. Fifteen treatment sessions will be performed over a three-week period. The primary outcomes will be measured by changes in the Numerical Rating Scale (NRS) scores and equivalent dosage of morphine at baseline, three weeks of treatment and one two-week follow-up. The secondary outcome measures include cellular immunity function, life quality assessment, opioids side effects assessment, and safety and compliance evaluation.

(Continued on next page)
\end{abstract}

\footnotetext{
* Correspondence: fangjianqiao7532@163.com

†Yi Liang and Guanai Bao contributed equally to this work.

'Department of Acupuncture, The Third Affiliated Hospital of Zhejiang

Chinese Medical University, No. 219 Moganshan Road, XiHu District,

Hangzhou 310005, Zhejiang Province, China

${ }^{2}$ The Third Clinical Medical College of Zhejiang Chinese Medical University,

No. 548 Binwen Road, Binjiang District, Hangzhou 310053, Zhejiang Province,

China

Full list of author information is available at the end of the article
}

(c) The Author(s). 2019 Open Access This article is distributed under the terms of the Creative Commons Attribution 4.0 International License (http://creativecommons.org/licenses/by/4.0/) which permits unrestricted use, distribution, and reproduction in any medium, provided you give appropriate credit to the original author(s) and the source, provide a link to the Creative Commons license, and indicate if changes were made. The Creative Commons Public Domain Dedication waiver (http://creativecommons.org/publicdomain/zero/1.0/) applies to the data made available in this article, unless otherwise stated. 
(Continued from previous page)

Discussion: This trial is expected to clarify whether TEAS is effective for cancer-related pain. These results demonstrate the advantage of TEAS combined with opioid drugs on improving immune function and decreasing opioid induced side effects.

Trial registration: Chinese Clinical Trial Registry, ChiCTR-13003803. Registered on 27 August 2013.

Keywords: Study protocol, Randomized controlled trial, TEAS, Cancer-related pain,

\section{Background}

Cancer-related pain, which always manifests as severe and intractable pain, is the most disruptive cancer-related event to the cancer patient's quality of life $[1,2]$. The overall prevalence of pain in patients with cancer had been reported as $>50 \%$ [2]. In a 2014 systematic review of 19 studies, the pooled prevalence of breakthrough cancer-related pain was $59.2 \%$, which ranged from $39.9 \%$ in outpatient clinics to $80.5 \%$ in hospices [3]. A Cochrane systematic review published in 2015 reported that $40 \%$ of patients with early or intermediate stage cancer and $90 \%$ with advanced cancer suffer from moderate or severe pain [4]. Although the World Health Organization (WHO) has given more attention to control cancer pain for several decades, under-treatment of cancer pain has been widely documented [5]. To date, opiate drugs remain the gold standard for treating moderate to severe pain or breakthrough pain resulting from cancer and are recommended strongly for treating cancer pain [6]. Nevertheless, long-term use of opioid drugs extensively inhibits the immune system [7] by leading to $\mathrm{T}$ lymphocyte apoptosis [8], inhibiting activation of $\mathrm{T}$ lymphocyte proliferation and secretion of IL-2 $[9,10]$. Opioid-induced immunosuppression has become the most significant drug-induced medical problem or side effect of opiate drug administration [11]. In addition, chronic pain (including cancer pain) also has an inhibitory effect on the immune system [12]. At present, an overwhelming majority of cancer patients are simultaneously bothered by both cancer-related pain and opioid-induced immunosuppression.

Acupuncture has been accepted worldwide given its effectiveness in treating various pains. A systematic review published recently demonstrated that acupuncture is effective in relieving cancer-related pain, particularly malignancy-related and surgery-induced pain [13]. Acupuncture plus drug therapy is more effective than conventional drug therapy alone for cancer-related pain [14]. Transcutaneous electrical nerve stimulation (TENS) has been widely used in acute or chronic pain, is effective in $67 \%$ of different types of pain [15], and may be a novel treatment for cancer bone pain [16]. However, the analgesic effects of TENS for chronic pain or cancer pain are obscure due to a lack of suitable randomized controlled trials (RCTs) $[17,18]$. Transcutaneous electrical acupoint stimulation (TEAS), also called acupuncture-like TENS or acupuncture-type TENS, is a novel therapy combined acupoint stimulation and TENS technique. TEAS has become more popular than acupuncture worldwide given its non-invasive feature. Moreover, it has been reported that the analgesic effect of TEAS was similar to acupuncture on postoperative surgical pain relief in gynecologic oncology patients [19]. Previous clinical studies also demonstrated a positive effect of TEAS analgesia in patients with labor pain and postoperative pain [20-23]. However, there is no direct evidence on the effect of TEAS on cancer-related pain. Moreover, acupuncture and TEAS has immunomodulatory effects $[24,25]$, but whether TEAS combined with opioid drugs will helpful to improve immune function and reduce other opioid-related side effects remains unclear. Rigorously designed and large multicenter RCTs are required to assess the value and potential advantage of TEAS in the management of cancer-related pain.

\section{Methods}

\section{Study design}

This multicenter, randomized controlled clinical trial will be performed in two inpatient units and comprises two parallel groups. After the inclusion and consent of the Institutional Review Board, participants will be randomized to either the control (sham TEAS) or TEAS group. The TEAS group will receive conventional cancer therapy (including 21-day interval chemotherapy and three steps analgesic therapy) and 60 additional minutes of TEAS treatment (30 min in each) five days per week for three weeks (15 sessions in total) during the inpatient stay. The control group will receive conventional cancer therapy and sham TEAS (lead wires of the apparatus will be cut down). The trial design is summarized in Fig. 1. The Standard Protocol Items: Recommendations for Interventional Trials (SPIRIT) checklist is provided as Additional file 1.

In all groups, participants will be permitted to use necessary analgesics such as short-acting morphine tablets or morphine injection during breakthrough cancer pain. The type, dose, and time of administration of the agent must be recorded in a cancer pain diary. The trial was registered in ClinicalTrials.gov with approval number ChiCTR-TRC-13003803.

\section{Participant recruitment}

A total of 160 participants will be recruited in the Zhejiang Cancer Hospital and the Third Affiliated Hospital 


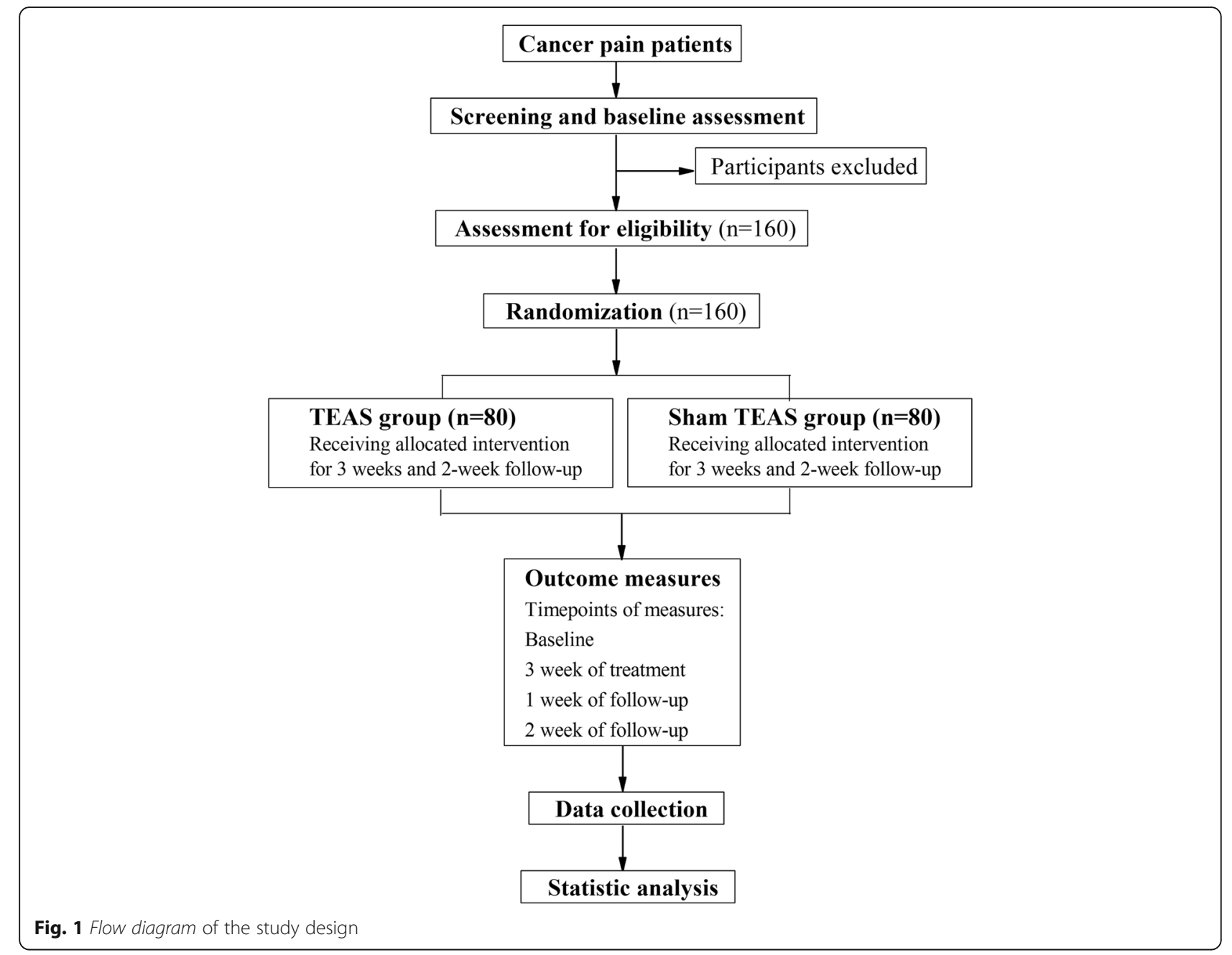

of Zhejiang Chinese Medicine University in Zhejiang Province, China. Our study will be advertised on the Internet and on posters in communities and hospitals. Prospective participants will be informed of the benefit and possible risk associated with this study. Participants will be told that they can withdraw from the trial at any time without specifying reasons. Participants will voluntarily provide written informed consent before enrollment. If prospective participants are interested in participating, they will be invited for a series of assessments by an oncologist. Eligible participants will be randomized into two groups with different treatments once informed consent has been obtained.

\section{Inclusion criteria}

Eligible participants should match the diagnostic criteria for cancer established by the Union for International Cancer Control (UICC) accompanied with pain induced by primary cancer, such as lung cancer, esophageal cancer, mammary cancer, gastric cancer, and pancreatic cancer. Patients must also meet the following criteria: (1) men or women aged 18-70 years with a $>2$-month expectant lifespan; (2) a numerical rating scale (NRS) score $\geq 4$ or treated by opioid drug therapy; (3) a Karnofsy performance state scale (KPS) score $\geq 60$; (4) receiving 21-day intervals of chemotherapy; (5) patients have the ability to estimate themselves (pain, quality of life, etc.); and (6) written informed consent was provided by themselves or their lineal kin.

\section{Exclusion criteria}

Patients receiving other analgesic therapy, such as radiation, bone cement, and nerve block therapy, with pain unrelated to cancer, will not be included. Patients who suffer from active cerebrovascular disease, respiratory depression, severe cognitive impairment, or mental disorders will not be included. Patients who are allergic to opioid drugs and patients with skin lesions in local acupoints will also be excluded.

\section{Ethical considerations}

The protocol of this study was approved by the ethics committees of the Third Affiliated Hospital of Zhejiang 
Chinese Medical University (permission number: ZSLL-KY-2013-016) and Zhejiang Cancer Hospital (permission number: zjzlyy [2014]-09-86号). The purpose, nature, and potential risks of this study were fully explained to the participants and their families. All participants have provided written informed consent for inclusion in this study.

\section{Randomization and blinding}

To guarantee allocation concealment, randomization will be performed by independent research staff. The random number generated by the SPSS statistical package program (version 20.0, SPSS, Inc., Chicago, IL, USA), and the treatment codes will be placed in sealed opaque envelopes. The study coordinator who does not participate in treatment or nursing was responsible for allocating the randomization codes. In this study, the outcome assessors, doctors-in-charge, data analysts, and participants will be blinded to the group assignments. However, it will be impossible to blind the TEAS therapist because they must be trained to perform the TEAS according to the research plan.

\section{Interventions}

The study is a randomized clinical trial that will be performed in the inpatient oncology ward of two hospitals. Participants will be randomized to either the control or the TEAS group. Both groups will receive 21-days interval chemotherapy according to the NCCN Clinical Practice Guidelines in Oncology (Version 2.2011, details in Table 1) and conventional cancer pain therapy under the guidance of the WHO three-step analgesic ladder principle. The TEAS group will receive 15 additional TEAS sessions, whereas the control group will receive sham stimulation of TEAS during the trial period.

\section{TEAS group}

The TEAS treatment strategy is designed by an experienced acupuncturist with $>30$ years of practice experience in acupuncture analgesia. TEAS will be performed by two doctors from the acupuncture ward who have a master's degree. All oncologists who enroll participants and the acupuncturist who manipulate TEAS will be trained together to ensure identical manipulation. The treatment will be started after randomization.

Participants in this group will receive 15 TEAS sessions in total over a three-week period. TEAS stimulation will be performed using a HANS Acupuncture Point Nerve Stimulator (HANS-100, Huawei Co., Ltd., Beijing, China) through connecting the square-shaped electrode patch $(3 \times 3 \mathrm{~cm})$ to the acupoint skin. Then, TEAS stimulation will be applied to L14 (He Gu) and PC6 (Nei Guan), ST36 ( Zu San Li) and SP6 (San Yin Jiao) for $30 \mathrm{~min}$ each time twice a day for five continuous days followed by a two-day rest interval. The two acupoint groups of the left side will be stimulated in the morning (09:00-11:00); those of the right side will be stimulated in the afternoon (14:00-16:00). The TEAS parameters are set as follows: dilatational square wave current output with $2 \mathrm{~Hz}$ and $100 \mathrm{~Hz}$ alternative frequency (pulse width: $0.6 \mathrm{~ms} / 0.2 \mathrm{~ms}$ ); intensities in the range of $8-12 \mathrm{~mA}$ according to each individual.

\section{Sham TEAS group}

Participants in the sham TEAS group, which serves as a control group, will also receive 21 days of interval chemotherapy and conventional cancer pain therapy; these therapies are the same as those in the TEAS group. Sham TEAS therapy (lead wires of the apparatus will be cut down) will provided during the trial period. Each step of the sham TEAS manipulation is same as real TEAS without electrical stimulation.

\section{Outcome measurement}

The patients will be carefully examined at baseline and reexamined after three weeks of treatment. Follow-up will be performed one and two weeks after completion of the TEAS treatment. Detailed time points of outcome assessments are provided in Fig. 2.

\section{Baseline assessments}

Baseline assessments will be conducted before randomization, including demographic characteristics (gender, age, height, weight, profession, and education levels of patients, diagnosis, stage of disease, and chemotherapy regimens), pain, equivalent dosage of morphine, cellular immunity function, and life quality.

\section{Primary outcome measures}

The primary outcome measure in the study is pain assessment demonstrated by Numerical Rating Scale (NRS) scores and equivalent dosage of morphine assessment. The dosage of opioid drugs should be converted to a morphine-equivalent dose according to NCCN Clinical Practice Guidelines in Oncology (Version 2.2011) and the following formulas: transdermal fentanyl $(25 \mathrm{mcg} / \mathrm{h}) \approx$ oral oxycodone $(30 \mathrm{mg} /$ d) $\approx$ parenteral morphine $(20 \mathrm{mg} /$ day $) \approx$ oral morphine (60 mg/day).

\section{Secondary outcome measures}

The secondary outcome measures include: (1) cellular immunity function: immune cell subsets distribution of all participants were detected using a Beckman Coulter FC500 flow cytometer before and after treatment; (2) the Karnofsky Performance Status Scale (KPS) and three-level EuroQol five-dimensional questionnaire (EQ-5D-3 L) for life quality assessment; (3) opioid side 
Table 1 Details of the 21-day interval chemotherapy regimen

\begin{tabular}{|c|c|c|c|c|}
\hline Chemotherapy regimen & Drugs & Dosage & Administration route & Medication time \\
\hline \multicolumn{5}{|l|}{ Combined chemotherapy } \\
\hline \multirow[t]{2}{*}{ NP } & Navelbine & $25 \mathrm{mg} / \mathrm{m}^{2}$ & i.v. & D1, D8 \\
\hline & Cis-platinum & $80 \mathrm{mg} / \mathrm{m}^{2}$ & i.v. & D1 \\
\hline \multirow[t]{3}{*}{$\mathrm{TP}$} & Paclitaxel & $135 \sim 175 \mathrm{mg} / \mathrm{m}^{2}$ & i.v. & D1 \\
\hline & Cis-platinum & $75 \mathrm{mg} / \mathrm{m}^{2}$ & i.v. & D1 \\
\hline & or Carboplatin & $A \cup C=5-6 \mathrm{mg} / \mathrm{m}^{2}$ & i.v. & D1 \\
\hline \multirow[t]{3}{*}{ GP } & Gemcitabine & $1250 \mathrm{mg} / \mathrm{m}^{2}$ & i.v. & D1, D8 \\
\hline & Cis-platinum & $75 \mathrm{mg} / \mathrm{m}^{2}$ & i.v. & D1 \\
\hline & or Carboplatin & $A \cup C=5-6 \mathrm{mg} / \mathrm{m}^{2}$ & i.v. & D1 \\
\hline \multirow[t]{3}{*}{ DP } & Docetaxel & $75 \mathrm{mg} / \mathrm{m}^{2}$ & i.v. & D1 \\
\hline & Cis-platinum & $75 \mathrm{mg} / \mathrm{m}^{2}$ & i.v. & D1 \\
\hline & or Carboplatin & $A \cup C=5-6 \mathrm{mg} / \mathrm{m}^{2}$ & i.v. & D1 \\
\hline \multirow[t]{3}{*}{ PC } & Pemetrexed & $500 \mathrm{mg} / \mathrm{m}^{2}$ & i.v. & D1 \\
\hline & Cis-platinum & $75 \mathrm{mg} / \mathrm{m}^{2}$ & i.v. & D1 \\
\hline & or Carboplatin & $\mathrm{AUC}=5-6 \mathrm{mg} / \mathrm{m}^{2}$ & i.v. & D1 \\
\hline \multirow[t]{3}{*}{ EP } & Etoposide & $100 \mathrm{mg} / \mathrm{m}^{2}$ & i.v. & D1-3 \\
\hline & Cis-platinum & $80 \mathrm{mg} / \mathrm{m}^{2}$ & i.v. & D1 \\
\hline & or Carboplatin & $A \cup C=5-6 \mathrm{mg} / \mathrm{m}^{2}$ & i.v. & D1 \\
\hline \multirow[t]{2}{*}{ EP } & Etoposide & $120 \mathrm{mg} / \mathrm{m}^{2}$ & i.v. & D1-3 \\
\hline & Cis-platinum & $60 \mathrm{mg} / \mathrm{m}^{2}$ & i.v. & D1 \\
\hline \multirow[t]{2}{*}{ IP } & Irinotecan & $65 \mathrm{mg} / \mathrm{m}^{2}$ & i.v. & D1, D8 \\
\hline & Cis-platinum & $30 \mathrm{mg} / \mathrm{m}^{2}$ & i.v. & D1, D8 \\
\hline \multirow[t]{2}{*}{ AP } & Amrubicin & $40 \mathrm{mg} / \mathrm{m}^{2}$ & i.v. & D1-3 \\
\hline & Cis-platinum & $60 \mathrm{mg} / \mathrm{m}^{2}$ & i.v. & D1 \\
\hline \multirow[t]{2}{*}{ XT } & Docetaxel & $75 \mathrm{mg} / \mathrm{m}^{2}$ & i.v. & D1 \\
\hline & Capecitabine & $950 \mathrm{mg} / \mathrm{m}^{2}$ & p.o. & D1-14 \\
\hline \multirow[t]{2}{*}{ GT } & Paclitaxel & $175 \mathrm{mg} / \mathrm{m}^{2}$ & i.v. & D1 \\
\hline & Gemcitabine & $1000 \sim 1250 \mathrm{mg} / \mathrm{m}^{2}$ & i.v. & D1, D8 \\
\hline \multirow[t]{2}{*}{ TC } & Docetaxel & $75 \mathrm{mg} / \mathrm{m}^{2}$ & i.v. & D1 \\
\hline & Cyclophosphamide & $600 \mathrm{mg} / \mathrm{m}^{2}$ & i.v. & D1 \\
\hline \multirow[t]{3}{*}{ TAC } & Docetaxel & $75 \mathrm{mg} / \mathrm{m}^{2}$ & i.v. & D1 \\
\hline & Doxorubicin & $50 \mathrm{mg} / \mathrm{m}^{2}$ & i.v. & D1 \\
\hline & Cyclophosphamide & $500 \mathrm{mg} / \mathrm{m}^{2}$ & i.v. & D1 \\
\hline \multicolumn{5}{|c|}{ Single-agent chemotherapy } \\
\hline Doxorubicin & Doxorubicin & $75 \mathrm{mg} / \mathrm{m}^{2}$ & i.v. & D1 \\
\hline Paclitaxel & Paclitaxel & $175 \mathrm{mg} / \mathrm{m}^{2}$ & i.v. & D1 \\
\hline Docetaxel & Docetaxel & $80 \mathrm{mg} / \mathrm{m}^{2}$ & i.v. & D1 \\
\hline Capecitabine & Capecitabine & $1000-1250 \mathrm{mg} / \mathrm{m}^{2}$ & p.o. & D1-14 \\
\hline Herceptin & Herceptin & $6 \mathrm{mg} / \mathrm{kg}$ & i.v. & D21 \\
\hline
\end{tabular}

i.v. intravenous injection, p.o. per os (oral administration)

effect assessment according to NCCN Clinical Practice Guidelines in Oncology (Version 2.2011); and (4) safety and compliance evaluation.

Any adverse events (AE) and serious AEs occurring during the trial will be recorded. AEs include fainting, severe pain, local infection, unbearable prickling during TEAS treatment, and nervous toxicity due to over-dosage of opioid drugs. All details, such as the date of occurrence, time, degree, and measurement related to the treatment will be documented.

\section{Quality control}

The trial protocol has been modified according to suggestions from experienced acupuncturists and oncologists. Before the trial, all researchers who enroll participants and collect data must attend a series of 


\begin{tabular}{|c|c|c|c|c|}
\hline Period & Baseline & Treatment phase & \multicolumn{2}{|c|}{ Follow-up phase } \\
\hline Visit & 1 & 2 & 3 & 4 \\
\hline $\begin{array}{c}\text { Week } \\
\text { (after randomization) }\end{array}$ & -1 & 1 to 3 & 4 & 5 \\
\hline Informed consent & $\times$ & & & \\
\hline Demographic characteristics & $\times$ & & & \\
\hline Inclusion/exclusion criteria & $\times$ & & & \\
\hline Random allocation & $\times$ & $\times$ & & \\
\hline TEAS & $\times$ & $\times$ & $\times$ & $\times$ \\
\hline Numerical rating scale (NRS) & $\times$ & $\times$ & & \\
\hline $\begin{array}{c}\text { Equivalent dosage of morphine } \\
\text { assessment (in the past 24 h) }\end{array}$ & $\times$ & $\times$ & $\times$ & $\times$ \\
\hline Cellular immunity assessment & $\times$ & $\times$ & & \\
\hline $\begin{array}{c}\text { Karnofsy performance state scale } \\
\text { (KPS) }\end{array}$ & $\times$ & $\times$ & \\
\hline $\begin{array}{c}\text { EuroQol five-dimensional } \\
\text { questionnaire (EQ-5D) }\end{array}$ & $\times$ & $\times$ & \\
\hline $\begin{array}{c}\text { Opioids side effects assessment } \\
\text { Safety evaluation }\end{array}$ & $\times$ & & & \\
\hline Compliance evaluation & $\times$ & & & \\
\hline
\end{tabular}

Fig. 2 Schedule of treatment and assessment. Outcomes at baseline will be assessed on the day before chemotherapy and TEAS stimulation; outcomes at treatment phase will be assessed on the day after the completion of TEAS or sham TEAS session; outcomes at follow-up phase will be evaluated at the last day of every period. $x=$ all groups; $(x)=$ TEAS groups

training sessions. These training sessions will ensure that the research staff involved fully understands the research protocol and standard operating procedures. During the trial, we will establish an inspector to guide and supervise the operators regularly (once every three months). In addition, unified production of various documents and materials, clear and detailed research plans, and assessment indicators and guides will be defined before and during the trial. Economic compensation and free TEAS treatment are also considered as methods for improving compliance. Data management and monitoring will be performed by using ResMan Research Manager (http://www.medresman.org). The principal investigator and the clinical epidemiologist will have access to all the data in the study.

\section{Sample size calculation}

The sample size of this study is estimated using the two proportions comparison method. According to our preliminary test, the average NRS score was decreased 0.051 in the TEAS group and increased 0.585 in the sham TEAS group. The combined standard deviation was 1.260 . A single-sided 5\% significance level and 90\% power were considered; the sample size will be calculated based on the equation below.

$$
n 1=n 2=\frac{2\left(u_{a}+u_{\beta}\right)^{2} \sigma^{2}}{\delta^{2}}
$$

Approximately 67 participants in each group were calculated to be required. Estimating a $20 \%$ dropout rate, 160 participants in total will be enrolled with 80 initial participants for each group.

\section{Statistical analysis}

All data in this study will be analyzed by a blinded statistician using the SPSS v20.0 (SPSS, Chicago, IL, USA). Independent sample $T$ test and Chi-square test ( $\chi^{2}$ test) will be used for numerical variables and categorical variables, respectively. When the distribution of variables is abnormal, a non-parametric test will be selected. A $P$ value $<0.05$ will be considered statistically significance.

\section{Discussion}

People worldwide are increasingly willing to accept acupuncture treatment given its therapeutic effect on analgesia. As novel types of acupuncture, TEAS or acupuncture-like TENS exert analgesic effects similar to acupuncture [19]. Moreover, TEAS becomes more acceptable than acupuncture given its non-invasive feature $[26,27]$. In recent years, research has increasingly 
focused on the analgesic effect of acupuncture on cancer-related pain. Given the lack of sufficient clinical evidence, whether acupuncture is effective for treating cancer pain in adults remains unclear [4]. Thus, further larger and methodologically sound trials are required [17]. In this trial, the therapeutic advantage of TEAS will also be assessed by investigating the changes on immunosuppression and other side effects result from opioid drugs in cancer pain patients. Cellular immune function will be detected regularly per routine practice in oncology wards, so investigating the immune-regulatory effect of TEAS is feasible.

Although each step of sham TEAS manipulation is the same as that in real TEAS with the exception of electrical stimulation (the lead wires will be cut down), blinding of TEAS treatment seems impossible because patients ultimately know whether they are receiving. To improve participant compliance, all patients enrolled in this trial will receive a 21-day interval of chemotherapy according to NCCN Clinical Practice Guidelines in Oncology and conventional cancer pain therapy under the guidance of the WHO three-step analgesic ladder principle. This process will improve participant compliance. Moreover, free TEAS treatment will further contribute to reducing the dropout rate.

Briefly, the purpose of this trial is to confirm whether TEAS is an effective adjunct to standard cancer pain therapy for moderate and severe cancer-related pain. This study may also confirm the advantage of TEAS on improving immune function, decreasing the dosage of opioid drugs and reducing the occurrence of opioid-related side effects.

\section{Trial status}

Participant enrollment started on 1 November 2014. Enrollment and trial completion are expected to be completed by the end of July 2018.

\section{Additional file}

Additional file 1: SPIRIT 2013 Checklist: Recommended items to address in a clinical trial protocol and related documents*. (PDF $182 \mathrm{~kb}$ )

\section{Abbreviations}

EQ-5D: EuroQol five-dimensional questionnaire; KPS: Karnofsy performance state scale; NCCN: National Comprehensive Cancer Network; NRS: Numerical rating scale; RCT: Randomized controlled trial; TEAS: Transcutaneous electrical acupoint stimulation; TENS: Transcutaneous electrical nerve stimulation; UICC: Union for International Cancer Control

\section{Acknowledgements}

We gratefully acknowledge all participants enrolled in this trial.

\section{Funding}

This study is supported by the National Natural Science Foundation of China (No. 81102643, 81674061) and the Foundation of the Zhejiang Health Committee (No. 2014KYA162). Sponsor and funders of this study do not have influence or authority regarding collection, management, analysis, and interpretation of data, writing of the report, or the decision to submit the report for publication.

\section{Authors' contributions}

$Y L, L Y G, X M G, W P Z$, and JQF participated in the conception and design of the trial. JZ, RR, GAB, XMS, YLJ, JYD, JFF, and NN participated in data collection and are in charge of patient recruitment. $\mathrm{CHJ}$ is planning the data analysis. YL, GAB, and NN drafted the manuscript. JQF and BYL revised the manuscript. All authors read and approved the final manuscript.

\section{Ethics approval and consent to participate}

The protocol of this study was approved by the ethics committees of the Third Affiliated Hospital of Zhejiang Chinese Medical University under the number ZSLL-KY-2013-016 and Zhejiang Cancer Hospital under the number zizlyy [2014]-09-86号. All patients signed the consent form to participate in this research.

\section{Consent for publication}

The authors agree to publish the present study protocol in this journal.

\section{Competing interests}

The authors declare that they have no competing interests.

\section{Publisher's Note}

Springer Nature remains neutral with regard to jurisdictional claims in published maps and institutional affiliations.

\section{Author details}

${ }^{1}$ Department of Acupuncture, The Third Affiliated Hospital of Zhejiang Chinese Medical University, No. 219 Moganshan Road, XiHu District, Hangzhou 310005, Zhejiang Province, China. ${ }^{2}$ The Third Clinical Medical College of Zhejiang Chinese Medical University, No. 548 Binwen Road, Binjiang District, Hangzhou 310053, Zhejiang Province, China. ${ }^{3}$ The Zhejiang Cancer Hospital, No. 1 Banshan East Road, Gongshu District, Hangzhou 310022, Zhejiang Province, China. ${ }^{4}$ The Clinical Research Institute of Zhejiang Provincial Hospital of TCM, No.54 Youdian Road, Xihu District, Hangzhou 310006, Zhejiang Province, China.

Received: 8 June 2018 Accepted: 17 December 2018

Published online: 11 January 2019

\section{References}

1. Neufeld NJ, Elnahal SM, Alvarez RH. Cancer pain: a review of epidemiology, clinical quality and value impact. Future Oncol. 2017;13(9):833-41.

2. van den Beuken-van Everdingen $M$, de Rijke J, Kessels $A$, Schouten $H$, van Kleef M, Patijn J. Prevalence of pain in patients with cancer: a systematic review of the past 40 years. Ann Oncol. 2007:18(9):1437-49.

3. Deandrea S, Corli O, Consonni D, Villani W, Greco MT, Apolone G. Prevalence of breakthrough cancer pain: a systematic review and a pooled analysis of published literature. J Pain Symptom Manag. 2014;47(1):57-76.

4. Paley CA, Johnson MI, Tashani OA, Bagnall AM. Acupuncture for cancer pain in adults. Cochrane Database Syst Rev. 2015;10:Cd007753.

5. Deandrea S, Montanari M, Moja L, Apolone G. Prevalence of undertreatment in cancer pain. A review of published literature. Ann Oncol. 2008;19(12): 1985-91.

6. Colson J, Koyyalagunta D, Falco FJ, Manchikanti L. A systematic review of observational studies on the effectiveness of opioid therapy for cancer pain. Pain Physician. 2011;14(2):E85-102.

7. Sacerdote P. Opioids and the immune system. Palliat Med. 2006;20(Suppl 1): s9-15.

8. $\quad$ Singhal P, Kapasi A, Reddy K, Franki N. Opiates promote T cell apoptosis through JNK and caspase pathway. Adv Exp Med Biol. 2001;493:127-35.

9. Sacerdote P, Bianchi M, Gaspani L, Manfredi B, Maucione A, Terno G, et al. The effects of tramadol and morphine on immune responses and pain after surgery in cancer patients. Anesth Analg. 2000;90(6):1411-4.

10. Houghtling RA, Mellon RD, Tan RJ, Bayer BM. Acute effects of morphine on blood lymphocyte proliferation and plasma IL-6 levels. Ann N Y Acad Sci. 2000;917:771-7

11. Wei G, Moss J, Yuan CS. Opioid-induced immunosuppression: is it centrally mediated or peripherally mediated? Biochem Pharmacol. 2003;65(11):1761-6.

12. Page GG, Ben-Eliyahu S. The immune-suppressive nature of pain. Semin Oncol Nurs. 1997;13(1):10-5. 
13. Chiu HY, Hsieh YJ, Tsai PS. Systematic review and meta-analysis of acupuncture to reduce cancer-related pain. Eur J Cancer Care. 2017;26(2): e12457.

14. Hu C, Zhang H, Wu W, Yu W, Li Y, Bai J, et al. Acupuncture for pain management in cancer: a systematic review and meta-analysis. Evid-Based Complement Altern Med. 2016;2016:1720239.

15. Johnson MI, Bjordal JM. Transcutaneous electrical nerve stimulation for the management of painful conditions: focus on neuropathic pain. Expert Rev Neurother. 2011;11(5):735-53.

16. Searle RD, Bennett MI, Johnson Ml, Callin S, Radford H. Transcutaneous electrical nerve stimulation (TENS) for cancer bone pain. J Pain Symptom Manag. 2009;37(3):424-8

17. Hurlow A, Bennett Ml, Robb KA, Johnson MI, Simpson KH, Oxberry SG Transcutaneous electric nerve stimulation (TENS) for cancer pain in adults. Cochrane Database Syst Rev. 2012:3:Cd006276.

18. Robb K, Oxberry SG, Bennett MI, Johnson MI, Simpson KH, Searle RD. A cochrane systematic review of transcutaneous electrical nerve stimulation for cancer pain. J Pain Symptom Manag. 2009;37(4):746-53.

19. Gavronsky S, Koeniger-Donohue R, Steller J, Hawkins JW. Postoperative pain: acupuncture versus percutaneous electrical nerve stimulation. Pain Manag Nurs. 2012;13(3):150-6.

20. Chao AS, Chao A, Wang TH, Chang YC, Peng HH, Chang SD, et al. Pain relief by applying transcutaneous electrical nerve stimulation (TENS) on acupuncture points during the first stage of labor: a randomized doubleblind placebo-controlled trial. Pain. 2007;127(3):214-20.

21. Sun $K$, Xing T, Zhang F, Liu Y, Li W, Zhou Z, et al. Perioperative transcutaneous electrical acupoint stimulation for postoperative pain relief following laparoscopic surgery: a randomized controlled trial. Clin J Pain. 2017:33(4):340-7.

22. Peng T, Li XT, Zhou SF, Xiong Y, Kang Y, Cheng HD. Transcutaneous electrical nerve stimulation on acupoints relieves labor pain: a nonrandomized controlled study. Chinese J Integr Med. 2010;16(3):234-8.

23. Lan F, Ma YH, Xue JX, Wang TL, Ma DQ. Transcutaneous electrical nerve stimulation on acupoints reduces fentanyl requirement for postoperative pain relief after total hip arthroplasty in elderly patients. Minerva Anestesiol. 2012;78(8):887-95.

24. Cabioglu MT, Cetin BE. Acupuncture and immunomodulation. Am J Chinese Med. 2008;36(1):25-36.

25. Wu H, Wang K, Li G, Meng D, Han J, Wang G, et al. Effects of transcutaneous acupoint electrical stimulation on the imbalance of Th1, Th2, Th17 and Treg cells following thoracotomy of patients with lung cancer. Exp Therapeutic Med. 2016;11(2):495-502.

26. Melzack R, Wall PD. Acupuncture and transcutaneous electrical nerve stimulation. Postgrad Med J. 1984;60:893-6.

27. Chesterton LS, van der Windt DA, Sim J, Lewis M, Mallen CD, Mason EE, et al. Transcutaneous electrical nerve stimulation for the management of tennis elbow: a pragmatic randomized controlled trial: the TATE trial. BMC Musculoskelet Disord. 2009:10:156.

Ready to submit your research? Choose BMC and benefit from:

- fast, convenient online submission

- thorough peer review by experienced researchers in your field

- rapid publication on acceptance

- support for research data, including large and complex data types

- gold Open Access which fosters wider collaboration and increased citations

- maximum visibility for your research: over $100 \mathrm{M}$ website views per year

At $\mathrm{BMC}$, research is always in progress.

Learn more biomedcentral.com/submissions 